\title{
A cytoplasmically inherited mutation in the fungus Phycomyces blakesleeanus
}

\author{
José Arnau, $†$ Francisco J. Murillo and Santiago Torres-Martínez* \\ Departamento de Genética y Microbiología, Facultad de Biología, Universidad de Murcia, 30071 Murcia, Spain
}

(Received 25 January 1990; revised 5 April 1990; accepted 25 April 1990)

\begin{abstract}
Fourteen mutants of the fungus Phycomyces blakesleeanus, showing high levels of resistance to copper, were isolated. In all the mutants, copper resistance behaved as a very variable and unstable trait. In the mutant strain MU102, the mutation was demonstrated to be cytoplasmically inherited. In addition, this mutant strain differed from the wild-type in growth, respiration rate, and shape and viability of spores.
\end{abstract}

\section{Introduction}

The fungus Phycomyces blakesleeanus has received considerable attention, mainly for the behavioural responses of its giant sporangiophores, the synthesis and regulation of carotenes, sexual differentiation, and the regulation of the dormancy and germination of spores [see Cerdá-Olmedo \& Lipson (1987) for a recent review on Phycomyces]. A genetic approach to these problems has been possible after the isolation of a large number of mutants. Almost all mutations described in P. blakesleeanus, however, define nuclear functions; the information about cytoplasmic inheritance is very scarce. Unlike other filamentous fungi (e.g. Fincham et al., 1979), only one case of a cytoplasmic mutation, affecting the normal accumulation of $\beta$-carotene, has been described for $P$. blakesleeanus (De la Concha \& Murillo, 1984).

In this paper we report the isolation and characterization of a copper-resistant mutant harbouring a cytoplasmically inherited mutation. The properties of this mutant are rather similar to those of well-characterized cytoplasmic mutants of some filamentous fungi, such as Neurospora and Aspergillus (Fincham et al., 1979), and it is the first report of a respiratory-deficient mutant in $P$. blakesleeanus.

† Present address: Chr. Hansen's Laboratorium A/S, 10-12 Boge Alle, PO Box 407, DK-2970 Horsholm, Denmark.

Abbreviations: NQO, 4-nitroquinoline-1-oxide; SHAM, salicylhydroxamic acid.

\section{Methods}

Strains and growth. The strains of P. blakesleeanus used in this work were the wild-type NRRL 1555 and the mutant S102, an auxotroph for nicotinic acid (Medina, 1977); the mutant strains that we obtained are described in Results. Culture media used (Sutter, 1975) were SIV, a glucose/asparagine minimal medium, and SIVY, which is a minimal medium supplemented with $1 \mathrm{mg}$ yeast extract $\mathrm{ml}^{-1}$. For colony growth, SIV and SIVY were acidified to pH 3.3 (SIVA and SIVYA, respectively). When needed, minimal medium was supplemented with $1 \mu \mathrm{g}$ nicotinic acid $\mathrm{ml}^{-1}$. To select for copper-resistance, media were supplemented, after autoclaving, with copper sulphate to the required concentration.

Cultures were initiated by activated asexual spores $\left(15 \mathrm{~min}\right.$ at $\left.48^{\circ} \mathrm{C}\right)$ or by a small piece of young mycelium, and incubated at 22 or $26^{\circ} \mathrm{C}$. Heterokaryons were produced by following the method of Ootaki $e t$ al. (1973).

Mutagenesis and selection for copper-resistance. All mutants described in this work were obtained after treatment of NRRL 1555 wild-type spores with 4-nitroquinoline-1-oxide (NQO) (Pharmaceuticals Inc), following the method described by Revuelta \& Eslava (1983).

Mutagenized spores were plated out on non-selective SIVY medium and incubated at $26^{\circ} \mathrm{C}$ for $5-7 \mathrm{~d}$. New spores were then harvested and subsequently inoculated on SIVA medium supplemented with $5 \mathrm{mM}$ copper sulphate and overlaid with a cellophane membrane. After $48 \mathrm{~h}$, the membrane was transplanted to fresh SIVA medium to allow the colonies to grow faster. Spores collected from individual colonies were recycled in the same way several times, and were finally inoculated in SIVA medium supplemented with copper sulphate, without a cellophane membrane. Colonies which grew faster in these conditions were then selected and copper-resistance levels were analysed by plating spores on selective and non-selective SIVA medium.

Respiratory activity. Respiratory activity was measured by using an oxygen polarograph (Yellow Springs Instruments, model 65) with a Clark electrode connected to a Hitachi register.

Spores from the wild-type and from the mutant strain MU102 were inoculated in liquid SIVY medium at $26^{\circ} \mathrm{C}$ for $7 \mathrm{~h}$. Germlings were 
then centrifuged and resuspended in $5 \mathrm{ml} 10 \mathrm{~mm}$-sodium phosphate buffer, $\mathrm{pH} 6 \cdot 5$, containing $10 \mathrm{~mm}-\mathrm{MgCl}_{2}$ and $2 \%(\mathrm{w} / \mathrm{v})$ glucose. This mixture was saturated with $\mathrm{O}_{2}$ by air bubbling; the $\mathrm{O}_{2}$ concentration of the saturated mixture was shown to be $270 \mu \mathrm{M}$. After several minutes, potassium cyanide and salicylhydroxamic acid (SHAM) were added to a final concentration of $1 \mathrm{mM}$

Carotene analysis. For carotene analysis, 7-d-old mycelia growing in solid medium were scraped off with a spatula, carefully cleaned to remove bits of agar, stored at $-30^{\circ} \mathrm{C}$, and lyophilized. Carotene extraction (De la Guardia et al., 1971) and chromatographic separation and identification (Ootaki et al., 1973; Davies, 1965) were as previously described.

\section{Results}

The effect of copper-sulphate on the growth of $P$. blakesleeanus wild-type spores is shown in Fig. 1. Survival of the spores decreased dramatically with increasing copper concentration, and no germination was detected at $5 \mathrm{~mm}$. This inhibitory concentration was used to select for copper-resistant mutants. Spores from two independent mutagenesis experiments with NQO were separately inoculated on several plates of nonselective medium. Then, spores from each plate were separately harvested and selected for copper resistance, as described in Methods. A single mutant was picked from each stock of selected spores. In this way, the probability of getting subclones of a single initial mutation was minimized. Fourteen mutants showing high levels of resistance to copper were isolated (Table 1). In all of them copper resistance behaved as a very variable and unstable trait, which was occasionally lost when the spores were repeatedly grown in non-selective medium (data not shown).

In addition to the high levels of copper resistance, all mutants had a low germination rate at $26^{\circ} \mathrm{C}$ (Table 1). This was also a very variable trait. When spores were collected from ten different colonies of mutant MU102, grown in non-selective medium, the mean viability was $0.98 \%$, with an SD of $\pm 0.8 \%$. In a similar experiment, the viability of wild-type spores was much more regular $(50 \cdot 2 \% \pm 2 \cdot 1 \%)$.

\section{Characterization of strain MU102}

Besides resistance to copper and a low germination rate, strain MU102 showed other phenotypic changes. Thus, a high proportion of the spores were not elliptical but were very irregular in shape and bring to mind early stages in protospore formation in P. blakesleeanus (Tu \& Malhotra, 1976). Also, the distribution of nuclei in the abnormal spore population of mutant MU102 was significantly different from that in wild-type spores, with a much higher mean number of nuclei in the mutant

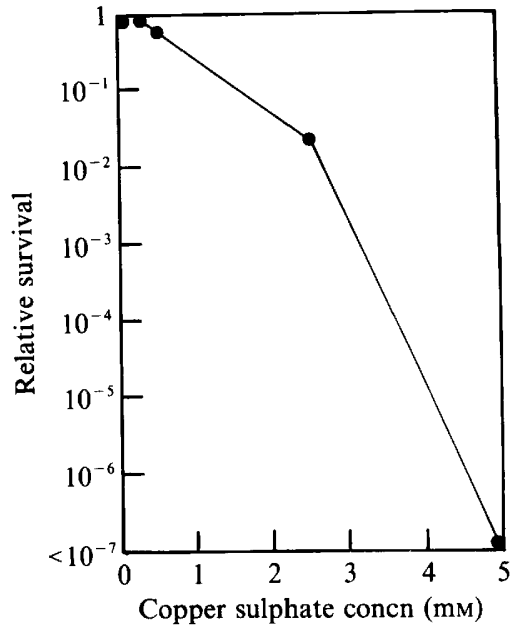

Fig. 1. Effect of different concentrations of copper sulphate on the viability of wild-type spores of $P$. blakesleeanus.

Table 1. Resistance to copper sulphate and spore viability of the Phycomyces mutants isolated in this work

\begin{tabular}{|c|c|c|}
\hline Strain & Survival* & Viability $\dagger$ \\
\hline Wild-type & $<10^{-4}$ & 51 \\
\hline MU102 & $15 \cdot 0$ & 0.07 \\
\hline MU111 & $3 \cdot 3$ & $1 \cdot 40$ \\
\hline MU112 & $2 \cdot 3$ & 0.26 \\
\hline MU120 & $2 \cdot 0$ & 2.65 \\
\hline MU121 & $2 \cdot 7$ & 8.05 \\
\hline MU122 & $5 \cdot 2$ & 1.74 \\
\hline MU123 & $9 \cdot 6$ & 0.08 \\
\hline MU124 & 1.4 & 0.07 \\
\hline MU125 & $2 \cdot 2$ & 0.04 \\
\hline MU127 & 1.4 & 0.13 \\
\hline MU129 & $2 \cdot 2$ & $7 \cdot 30$ \\
\hline MU130 & $2 \cdot 0$ & 1.00 \\
\hline MU131 & $2 \cdot 9$ & 1.52 \\
\hline MU133 & $8 \cdot 5$ & $8 \cdot 66$ \\
\hline
\end{tabular}

* Percentage of colonies formed in SIVA plates containing $5 \mathrm{~mm}$-copper sulphate, relative to the number formed in SIVA plates.

+ Percentage of germinated cells at $26^{\circ} \mathrm{C}$ in SIVA plates, relative to the number of spores observed by microscopy.

than in the wild-type. More than 100 spores were screened, both for the mutant and the wild-type strain: the mean number of nuclei in the mutant spores was 9.42 $(\mathrm{SD}=2.57)$ compared with $3.79(\mathrm{SD}=0.91)$ for the wildtype. The MU102 mutant also showed defective growth, with patches of mycelium with poor production of sporangiophores, occasionally accompanied by abnormal pigmentation due to the accumulation of a mixture of intermediates of the $\beta$-carotene pathway. Phytofluene, $\zeta$-carotene, neurosporene and $\beta$-carotene were easily identified after chromatographic separation of the carotenes accumulated (Fig. 2). The presence of carotenes other than $\beta$-carotene, the only pigment present in 


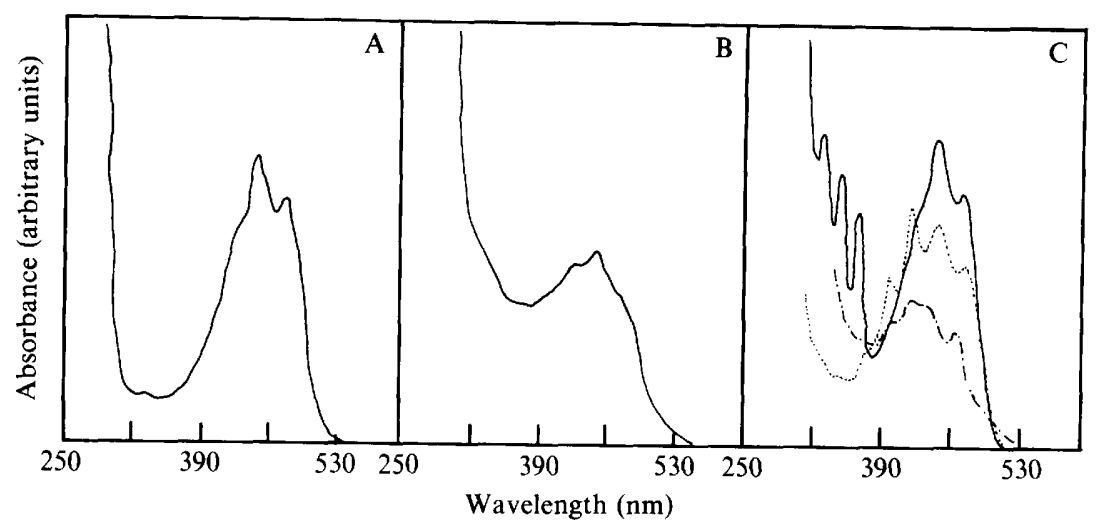

Fig. 2. Spectra of total carotenes accumulated in (A) NRRL 1555 and (B) mutant strain MU102. (C) Spectra of the carotenes accumulated in strain MU102, successively eluted with light petroleum $(-; \cdots)$ and acetone (-.---) (see Methods).

the wild-type strain of $P$. blakesleeanus, suggests that the mutation present in strain MU102 affects the normal accumulation of pigments.

As stated above, the phenotype of MU102 and other copper-resistant mutants was an unstable trait, growth in non-selective media generating copper-sensitive spores. Several such copper-sensitive colonies derived from MU102 were analysed; they all showed wild-type phenotype for both carotenogenesis and spore size and shape.

\section{Respiratory activity}

Respiratory activity of mutant MU102 with glucose as substrate was variable but always lower than that of the wild-type strain. Differences between wild-type and mutant strains were also observed when sensitivity of respiration to cyanide and SHAM was measured. As described by Van Laere et al. (1980), spores of $P$. blakesleeanus have both normal cyanide-sensitive oxidation and an alternative electron transport pathway insensitive to cyanide but sensitive to SHAM. Fig. 3 shows measurements of the respiratory activity of germinated spores of the wild-type and of mutant MU102, as well as their sensitivity to inhibitors. Respiration in the wild-type germlings was completely resistant to SHAM and partially inhibited by cyanide. However, respiratory activity was completely inhibited after the addition of both inhibitors. On the other hand, respiration in the mutant was completely inhibited by SHAM alone but not by cyanide, suggesting that the normal cyanide-sensitive pathway is not operative in this strain.

\section{Heterokaryon test}

Since the behaviour of MU102 partially resembled that of some well-characterized cytoplasmic mutants of other

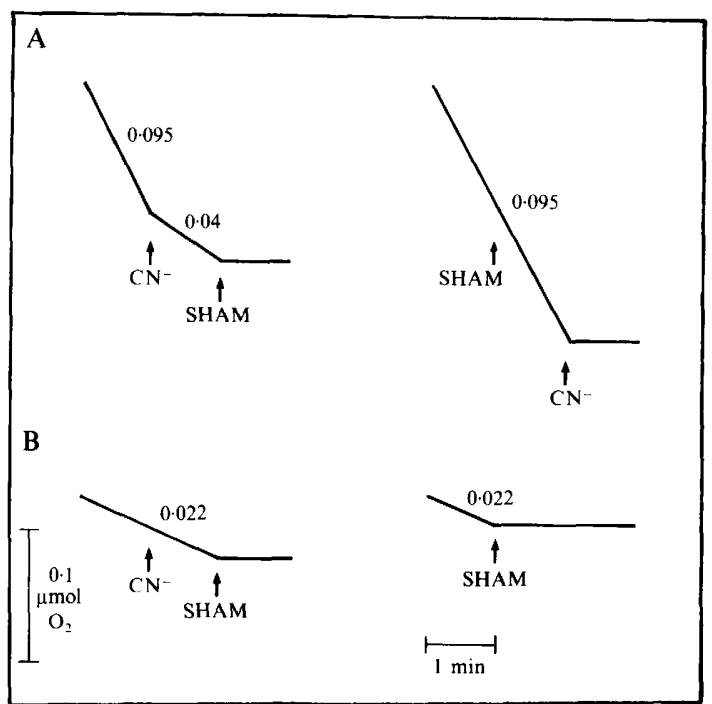

Fig. 3. Effect of cyanide and SHAM on respiratory activity of (A) wild-type strain NRRL 1555 and (B) mutant strain MU102. Respiratory activity of germling suspensions was monitored as described in the text. The respiratory inhibitors $\mathrm{CN}^{-}(1 \mathrm{~mm})$ and SHAM (1 mM) were added as indicated. Numbers indicate $\mu \mathrm{mol} \mathrm{O}_{2}$ consumed per minute per $5 \times 10^{6}$ germlings.

filamentous fungi, a 'heterokaryon test' (Jinks, 1958) was done using MU102 and S102; the latter strain harbours a nuclear recessive mutation leading to nicotinic acid auxotrophy (Medina, 1977). After screening 200 colonies from several heterokaryons, three $\mathrm{Nic}^{-}$, copper-resistant colonies were isolated, all of them showing, in addition, the other phenotypic traits of MU102. The rescue of the $\mathrm{Nic}^{-}$phenotype linked to the phenotypic features of MU102 is unequivocal evidence for the cytoplasmic nature of the mutation present in MU102. Otherwise, due to the recessive nature of the nic mutation it would not have been possible to rescue this mutation linked to copper-resistance after segregation of spores from the heterokaryons. 


\section{Discussion}

We report here the isolation and characterization of a copper-resistant mutant of $P$. blakesleeanus. In addition to the copper-resistance phenotype, this mutant also differs from the wild-type in growth, respiration rate, and shape and viability of spores. Some of these phenotypic traits show occasional vegetative segregation. In the absence of selective pressure, copper resistance and viability of spores from different colonies (data not shown) show great variability. This variability has usually been attributed to the unequal distribution of cytoplasmic elements during formation of the spores (Jinks, 1954, 1957). The occurrence of a heterogeneous population of cytoplasmic elements would also explain the loss of copper resistance observed when spores of strain MU102 are grown repeatedly in the absence of selective pressure.

The 'heterokaryon test' between strains MU102 and S102 clearly shows the cytoplasmic nature of the copperresistance trait and all other phenotypic traits of MU102. Furthermore, the defective respiratory activity of MU102 (Fig. 3) suggests a mitochondrial origin for the corresponding mutation. Copper is known to be a strong inhibitor of respiration in other organisms, such as Tetrahymena, acting preferentially on the synthesis of a number of mitochondrial components, including cytochromes (Wakatsuki et al., 1986).

The results in Fig. 3 support the presence of an 'alternative' respiratory pathway present alongside the main cyanide-sensitive cytochrome pathway, the alternative pathway being resistant to cyanide and sensitive to SHAM. Cyanide-resistant respiration has been reported in other filamentous fungi, such as Mucor (RuizHerrera et al., 1983) and Neurospora (Edwards \& Rosenberg, 1976; Lambowitz \& Slayman, 1971) and is widespread among plants (Siedow \& Berthold, 1986).

The cyanide-resistant respiration seems to be usually repressed in wild-type germlings of Phycomyces, since their respiration is not affected by SHAM, although, the contribution of the cyanide-resistant pathway may be masked by the main cyanide-sensitive one. However, after addition of cyanide to wild-type germlings, high respiratory activity is still observed (Fig. 3), suggesting that the cyanide-sensitive pathway must be blocked before the alternative one becomes active.

The behaviour of strain MU102 resembles that of cyanide-sensitive pathway mutants of Mucor (RuizHerrera et al., 1983) and Neurospora (Edwards \& Rosenberg, 1976; Lambowitz \& Slayman, 1971).

The pleiotropic effect of the MU102 mutation might be a consequence of the importance of normal mitochondrial function in the development of Phycomyces. Thus, spore formation, germination, vegetative growth and phorogenesis seem to be strongly dependent on the presence of functional mitochondria. The abnormal accumulation of intermediate substrates of $\beta$-carotene biosynthesis (Fig. 2) can be explained by assuming a mitochondrial membrane location for the multi-enzymic complex involved in $\beta$-carotene biosynthesis (De la Guardia et al., 1971; Aragón et al., 1976). A mitochondrial misfunction would affect the efficiency of substrate transfer along the carotene pathway.

The involvement of mitochondria in the biosynthesis of $\beta$-carotene is supported by another cytoplasmic mutation previously described in P. blakesleeanus (De la Concha \& Murillo, 1984). Under certain conditions, mutations in the car $E$ gene result in accumulation of $\beta$ carotene associated with proteins, in a red-complex form which is not found in the wild-type strain of $P$. blakesleeanus. This mutation behaves as an extranuclear genetic factor and there is some evidence for the presence of altered mitochondria in strains harbouring this mutation (A. De la Concha, personal communication).

The analysis of new mitochondrial mutants will be very useful for the study of those phenomena in which a functional mitochondrion is required. In that sense, selection for copper-resistant mutants seems to be a simple and valuable method to isolate strains of Phycomyces affected in mitochondrial functions.

\section{References}

Aragón, C. M. G., Murillo, F. J., De la Guardia, M. D. \& CerdáOLMEDO, E. (1976). An enzyme complex for the dehydrogenation of phytoene in Phycomyces. European Journal of Biochemistry 63, 71-75.

Cerdá-Olmedo, E. \& LiPson, E. (1987). Phycomyces. Cold Spring Harbor, NY: Cold Spring Harbor Laboratory.

Davies, B. H. (1965). Analysis of carotenoid pigments. In Chemistry and Biochemistry of Plant Pigments, pp. 489-532. Edited by T. W. Goodwin. London: Academic Press.

De la Concha, A. \& Murillo, F. J. (1984). Accumulation of a complex form of beta-carotene by Phycomyces blakesleeanus cytoplasmic mutants. Planta 161, 233-239.

De la Guardia, M. D., Aragón, C. M. G., Murillo, F. J. \& CerdáOlmedo, E. (1971). A carotenogenic enzyme aggregate in Phycomyces: evidence from quantitative complementation. Proceedings of the National Academy of Sciences of the United States of America 68, 2012-2015.

EDWARDS, D. L. \& Rosenberg, E. (1976). Regulation of cyanideinsensitive respiration in Neurospora. European Journal of Biochemistry 62, 217-221.

Fincham, J. R. S., DAY, P. R. \& RADFORD, A. (1979). Fungal Genetics. Oxford: Blackwell.

JINKS, J. L. (1954). Somatic selection in fungi. Nature, London 174, 409410.

Jinks, J. L. (1957). Selection for cytoplasmic differences. Proceedings of the Royal Society of London B146, 527-541.

JiNKs, J. L. (1958). Cytoplasmic differentiation in fungi. Proceedings of the Royal Society of London B148, 314-321. 
Lambowitz, A. M. \& Slayman, C. W. (1971). Cyanide-resistant respiration in Neurospora crassa. Journal of Bacteriology 108, 10871096.

MEdiNA, J. R. (1977). Continuous variation of genic dosage in Phycomyces. Genetical Research 30, 211-219.

Ootaki, T., Lightly, A. C., Delbrück, M. \& Hsu, W. J. (1973). Complementation between mutants of Phycomyces deficient with respect to carotenogenesis. Molecular and General Genetics 121, 5770.

Revuelta, J. L. \& Eslava, A. P. (1983). A new gene ( $c a r C$ ) involved in the regulation of carotenogenesis in Phycomyces. Molecular and General Genetics 192, 225-229.

Ruiz-Herrera, J., Ruiz, A. \& Lopez-Romero, E. (1983). Isolation and biochemical analysis of Mucor bacilliformis monomorphic mutants. Journal of Bacteriology 156, 264-272.
Siedow, J. N. \& BerTHOLD, D. A. (1986). The alternative oxidase: a cyanide-resistant respiratory pathway in higher plants. Physiologia Plantarum 66, 569-573.

SUTTER, R. P. (1975). Mutations affecting sexual development in Phycomyces blakesleeanus. Proceedings of the National Academy of Sciences of the United States of America 72, 127-130.

TU, J. C. \& MALHOTRA, S. K. (1976). The formation of sporangiospores in Phycomyces. Microbios 15, 15-25.

Van laere, A. J., Van Assche, J. A. \& Carlier, A. R. (1980). Metabolism and chemical activation of Phycomyces blakesleeanus spores. Experimental Mycology 4, 96-104.

WaKaTsukI, T., YohichI, T. \& HiRotsugu, I. (1986). Respiratory inhibition by copper in Tetrahymena pyriformis. Journal of Fermentation Technology 64, 119-128. 